\title{
A Program to Research Emittance Growth in Bends
}

\author{
Courtlandt L. Bohn \\ Continuous Electron Beam Accelerator Facility \\ Newport News, VA 29606
}

\begin{abstract}
A research program to explore the phenomenon of emittance growth in bends due to noninertial space-charge effects has been defined and initiated. The program combines theoretical, numerical, and experimental investigations. This paper summarizes the motivation of the work and highlights CEBAF's need for immediate results. The program's key elements, some of which qualitatively differ from the standard approach used to investigate the production and effects of coherent synchrotron radiation in synchrotrons and storage rings, are enumerated and discussed.
\end{abstract}

\section{INTRODUCTION}

The generic problem of emittance growth in bends due to noninertial space-charge effects and coherent synchrotron radiation has been circulating about the accelerator community for the past decade, mostly in conjunction with the prospect of producing high-brightness beams (1). The bulk of the most recent activities have centered on calculating the associated longitudinal wakefields in an effort to determine how short a bunch can be maintained in storage rings which are designed to be sources of coherent synchrotron radiation (2). However, the problem is also of interest in connection with the design of recirculating linear accelerators for high-brightness beams, in which the recirculation arcs can trigger emittance growth and beam degradation.

CEBAF has designed a linac of this type to drive a high-power ultraviolet free-electron laser (UVFEL) for industrial use in exploring its applications for processing materials (3). The design incorporates $180^{\circ}$ recirculation arcs which return the beam to the linac for energy recovery. There is limited margin for transverse emittance growth in the accelerator, and emittance growth in bends, especially the recirculation bends, is of paramount concern. We therefore have constructed a well-defined program to study the phenomenon and are seeking the means for its timely execution.

In what follows, the CEBAF UVFEL design is briefly described, the concern about emittance growth in the bends of this machine is explicitly identified,

\footnotetext{
${ }^{1}$ CEBAF is operated by the Southeastern Universities Research Association for the Department of Energy under Contract DE-AC05-84ER40150.
}

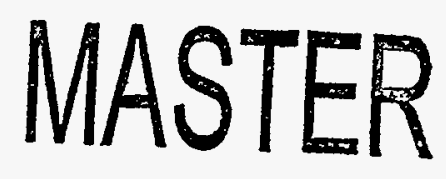




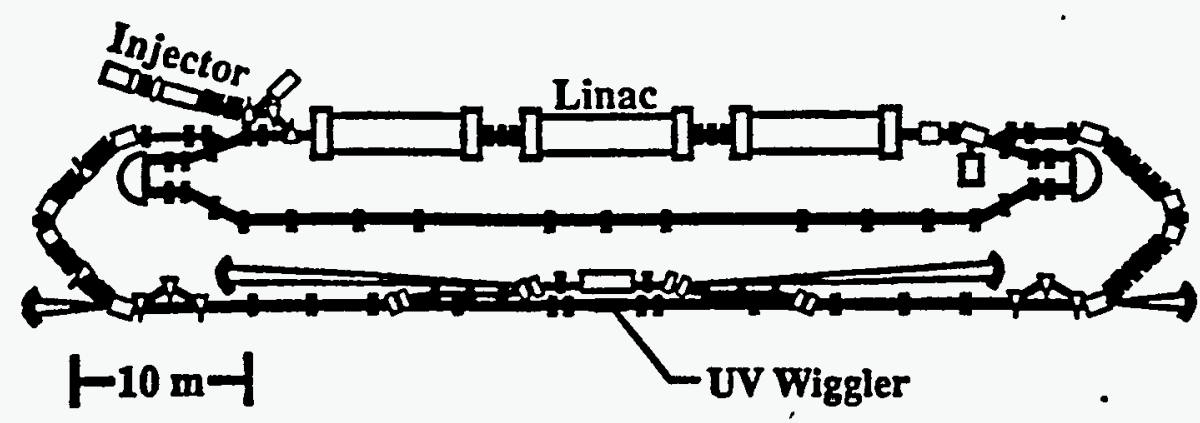

FIG. 1. Schematic of CEBAF's design of a UVFEL with a superconducting linac and recirculation for energy recovery. Two wigglers and optical resonators are shown, one for ultraviolet radiation, and one for infrared radiation.

and the program for systematically investigating the phenomenon is delineated.

\section{UVFEL DRIVER ACCELERATOR}

CEBAF has designed a recirculating, superconducting radiofrequency (SRF) accelerator with energy recovery to drive efficiently a high-power UVFEL designed to produce $1 \mathrm{~kW}$ continuous-wave laser light at wavelengths as short as $190 \mathrm{~nm}$. It is illustrated in Figure 1. The injector, which is now under construction, consists of a $500 \mathrm{kV}$ direct-current pholocathode gun and a CEBAF SRF quarter-cryomodule. It is designed to provide $10 \mathrm{MeV}$ beam of $5 \mathrm{~mA}$ average current with a normalized root-mean-square (rms) transverse emittance of nominally $5 \pi$-mm-mrad (4). The beam then passes through a sequence of three SRF cryomodules for acceleration to $106 \mathrm{MeV}$. Two $180^{\circ}$ achromatic, isochronous bends return the beam to the accelerator for boosting to $200 \mathrm{MeV}$. A $180^{\circ}$ nonisochronous reverse-bend outer arc compresses the bunch length from about 2 psec down to about $0.2 \mathrm{psec}$ and inserts the beam into the UV wiggler to produce the laser light. The wiggler spreads the bunch energy by a factor of about 5 , and this low-brightness beam is transported back through the accelerator twice for deceleration to $10 \mathrm{MeV}$, at which energy the beam is dumped. The residual beam energy is recycled to RF power and used to drive new beam.

Insofar as possible, the UVFEL was designed in a manner which spreads the risk across its constituent technologies (5). Its performance is most sensitive to the electron-beam energy $E_{b}$, the average current $\bar{I}$, and the normalized rms transverse emittance $\epsilon_{n}$ and it is relatively insensitive to the longitudinal 
phase-space properties of the beam. At wavelengths near $190 \mathrm{~nm}$, the output laser power varies approximately as $E_{b}^{3} \bar{I} / \epsilon_{n}$. To achieve $1 \mathrm{~kW}$ laser output at $190 \mathrm{~nm}$ requires $\epsilon_{n} \lesssim 11 \pi$-mm-mrad. Production of this beam clearly constitutes a high-brightness challenge.

\section{AN ESTIMATE OF EMITTANCE GROWTH IN THE UVFEL RECIRCULATION BENDS}

The bends comprising the inner arcs of the UVFEL are both isochronous and achromatic. In principle, an achromatic bend generates zero emittance growth in a beam bunch, even in the presence of an intrinsic energy spread. However, if an individual particle's energy changes while the bunch is transiting through the bend, then its final bend angle changes in proportion to the energy change. As pointed out in the recent work of Carlsten and Raubenheimer (6), hereafter called " $\mathrm{CR}$ ", this causes the rms transverse emittance to grow to a maximum value which is proportional to the rms energy change of the ensemble of particles comprising the bunch. Unlike emittance growth from inertial space-charge forces, which varies inversely with the square of the beam energy, this emittance growth is due to noninertial effects and is independent of the beam energy.

CR estimate that, with sufficiently short bunch lengths, the emittance growth is predominantly due to the longitudinal component of the Lorentz force acting on the bunches and is given by

$$
\Delta \epsilon_{n}=0.38 \alpha^{2} \frac{I_{p}}{I_{A}} \ln \left(\frac{\rho}{a}\right) \frac{a^{2}}{\delta},
$$

in which $\alpha$ is the bend angle in radians, $I_{p}$ is the peak current, $I_{A}$ is the Alfven current, $\rho$ is the radius of the beam pipe, $a=2 \tilde{x}$ is twice the rms transverse beam radius $\tilde{x}$, and $\delta$ is the bunch length, interpreted here as $\delta=6 \tilde{z}$ with $\tilde{z}$ denoting the rms bunch length. This emittance growth adds in quadrature with the input emittance. It is clearly a strong function of the bunch's aspect ratio, varying as $I_{p} a^{2} / \delta \propto(\tilde{x} / \tilde{z})^{2}$, and it is independent of the bend radius.

Nominal bunch parameters in the inner arcs of the UVFEL are $I_{p}=36 \mathrm{~A}$, $\rho=2.5 \mathrm{~cm}, a=0.8 \mathrm{~mm}$, and $\delta=2.7 \mathrm{~mm}$. The nominal beam size in the outer arc leading to the wiggler is $a=0.5 \mathrm{~mm}$, and representative average values for $I_{p}$ and $\delta$ through this arc are $72 \mathrm{~A}$ and $1.3 \mathrm{~mm}$, respectively. If all the bends are uncorrelated and the input emittance from the injector is $5 \pi$-mm-mrad, the estimated emittance growth from the CR formalism is about $16 \pi$-mm-mrad at the wiggler, which exceeds the maximum permissible emittance of $11 \pi$-mm-mrad.

The CR formalism is, however, only approximate and is based on perturbation theory for very short bunches. The dispersion relation for modes in the pipe is excluded. In addition, the electromagnetic fields acting on the bunch are calculated from a centrifugal space-charge potential which excludes 
the full effect of coherent synchrotron radiation (CSR). Thus, Carlsten and Raubenheimer believe their estimate constitutes a lower limit to the emittance growth (7).

Based on the dispersion relation for a toroidal chamber, one may expect CSR to be suppressed in a curved beam pipe if $\rho^{3} /\left(R \tilde{z}^{2}\right) \ll 1$, in which $R$ is the bend radius (1). In the inner-pass UVFEL bends, $R=1 \mathrm{~m}$ and this inequality is strongly violated, which means CSR will likely play a role.

Thus, notwithstanding the detailed bunch dynamics through the arcs, we are led to expect some degradation of emittance through the transport lattice of the UVFEL, and the degradation could conceivably be severe. If so, there are a number of possible cures, including: transverse focusing to reduce $a$, bunch lengthening to increase $\delta$ and/or lowering the bunch charge (while keeping $I_{p}$ large enough to generate high laser power), increasing $R / \rho^{3}$, using lossy pipe walls, or breaking each bend into a series of $N$ uncorrelated achromatic bends to reduce the emittance growth by $1 / N$. There is also a need to keep beam loss low throughout the machine, below $5 \mu \mathrm{A}$ at energies above $25 \mathrm{MeV}$. This introduces an additional concern about beam loss triggered by the electromagnetic fields in the pipe. The potential problems in the machine introduced by noninertial space-charge effects are clearly serious enough to warrant a program of expeditious research.

\section{THEORETICAL PROGRAM}

The electrodynamics of a single bunch transiting through a finite-length bend differs in a fundamental way from that of a bunch, or bunch train, circulating in a storage ring. In a bend, the electrodynamics necessarily encompasses transient phenomena, whereas in a storage ring, the electrodynamics will reach a steady state. This consideration applies, for example, in the UVFEL bends because the bunches are spaced far enough apart that the residual fields inside the bend will damp during the interim between successive bunch passages. Thus, a complete theory will need to include transients, and, because the temporal structure of the transients seen by particles in the bunch will be influenced by the reflective boundary of the conducting pipe, the theory will also have to incorporate the boundary conditions defined by the pipe walls.

A general prescription for a theory which does this involves solving Maxwell's equations for the electromagnetic fields while incorporating both the temporal and spatial boundary conditions. One approach for implementing this prescription is to use a model for the current density which is consistent with the continuity equation. This is the approach Warnock and Morton adopt to model a storage ring (1), but the temporal boundary conditions introduce additional terms to the fields which account for transients. Once the fields are known, the Lorentz force seen by individual particles comprising the bunch can be calculated. The particle orbits can then be determined and 
This report has been reproduced from the best available copy.

Available to DOE and DOE contractors from the Office of Scientific and Technical Information, P.O. Box 62, Oak Ridge, TN-37831; prices available from (615)576-8401, FTS 626-8401.

Available to the public from the National Technical Information Service, U.S. Department of Commerce, 5285 Port Royal Rd., Springfield, VA 22161.

\section{Price: Printed copy 402 Microfiche 101}


used to find the rms emittance growth. Moreover, the fields can be used also in a particle code to simulate the propagation of a bunch through the bend as part of a more careful analysis of emittance growth and beam scraping. Strictly speaking, however, this approach lacks self-consistency because it incorporates a "rigid-bunch approximation" through the model of the current density. Nevertheless, it provides a useful and relatively complete starting point for investigating the effect.

We have begun developing and testing this formalism in idealized geometries. Our plan is to apply it to the case of a pipe having the form of a toroidal sector, and then to the UVFEL bends.

\section{EXPERIMENTAL PROGRAM}

In view of the potential impact of noninertial effects on the UVFEL beam and the technical difficulties inherent in developing an accurate theory, we consider an experiment to be of utmost importance. Our current plan is to construct a scale model of one of the inner-pass bends in the machine, install it at the end of the $10 \mathrm{MeV}$ injector described above, and perform a series of experiments. The corresponding transport lattice has been identified, and its engineering design is about to begin. The essential measurements are envisioned to be the straight-line rms transverse emittance from the injector and the rms transverse emittance at the exit of the bend. Both the pipe size and injector beam properties would be varied to check scaling and to pass from the regime of full coherent synchrotron radiation (large pipe radius, short bunch) through suppression of this radiation (small pipe radius, long bunch).

There are two disadvantages to this plan. First, the beam energy is low and there is a risk that emittance growth from the energy-dependent space-charge force will comprise a large background for the measurement of the noninertial effect. An estimate with the CR formula suggests the noninertial space-charge effect is about five times larger than the conventional effect in this setup, but a wider margin would provide more confidence. Second, in view of the development schedule for the injector, the earliest the experiment could be done is summer 1996. In view of its importance, we would like to do the experiment sooner, and we are exploring the possibility of using existing facilities at other laboratories.

\section{CONCLUSION}

A study of noninertial space-charge effects and coherent synchrotron radiation in bends is of immediate practical importance for CEBAF's UVFEL driver accelerator. It is also of general importance with regard to the development of compact, high-brightness accelerators because their designs will likely incorporate magnetic bends for small machine footprints. We have set forth a 
plan for systematically investigating this problem on all fronts: theoretically, numerically, and experimentally. We want to do the experiment as early as possible and are exploring collaborations with other laboratories to facilitate this.

\section{ACKNOWLEDGEMENTS}

Several people at CEBAF are currently engaged in various aspects of this research. The ones who have been most helpful in identifying the fundamental issues and guiding principles are J. J. Bisognano and J. R. Delayen, and the author gratefully acknowledges their advice.

\section{REFERENCES}

1. See, for example, R. L. Warnock and P. Morton, Part. Accel. 25, 113 (1990) and references therein.

2. J. B. Murphy, S. Krinsky, and R. L. Gluckstern, "Longitudinal Wakefield for Synchrotron Radiation", Proceedings of the 1995 Particle Accelerator Conference, Dallas, Texas (in publication), and also related papers in this Microbunches Workshop Proceedings.

3. G. R. Neil, S.V. Benson, H. F. Dylla, and H. Liu, Nucl. Instr. and Meth. in Phys. Res. A358, 159 (1995).

4. H. Liu, this Proceedings.

5. "Free-Electron Lasers for Industry", CEBAF Report prepared for the Department of Energy UVFEL Conceptual Design Review, May 1995 (unpublished).

6. B. E. Carlsten and T. O. Raubenheimer, Phys. Rev. E 51, 1453, (1995).

7. T. O. Raubenheimer, private communication.

\section{DISCLAIMER}

This report was prepared as an account of work sponsored by an agency of the United States This report was prepared as anited States Government nor any agency thereof, nor any of their employees, makes any warranty, express or implied, or assumes any legal liability or responsibility for the accuracy, completeness, or usefulness of any information, apparatus, product, or process disclosed, or represents that its use would not infringe privately owned rights. Reference herein to any specific commercial product, process, or service by trade name, trademark, manufacturer, or otherwise does not necessarily constitute or imply its endorsement, recommanufacturer, or othing by the United States Government or any agency thereof. The views and opinions of authors expressed herein do not necessarily state or reflect those of the United States Government or any agency thereof. 
This report hes been reproduced from the best available copy.

Available to DOE and DOE contractora from the Office of Scientific and Technical Information, P.O. Box 62, Oak Ridge, TN-37831; prices available from (615)576-8401, FTS 626-8401.

Available to the public from the National Technical Information Service, U.S. Department of Commerce, 5285 Port Royal Rd., Springfield, VA 22161.

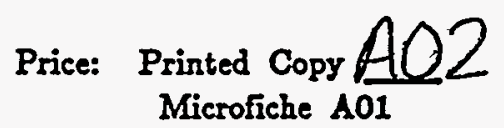




\section{DISCLAIMER}

Portions of this document may be illegible in electronic image products. Images are produced from the best available original document. 